\title{
A Fuzzy Logic based Recommender System for E- Learning System with Multi-Agent Framework
}

\author{
Himanshu Pandey \\ Department of Computer Science \\ B.N College of Engineering \& Technology \\ Lucknow, INDIA.
}

\author{
V. K Singh, PhD \\ Head- Department of Information Technology \\ BBDNITM \\ Lucknow, INDIA.
}

\begin{abstract}
In this paper a multi agent based e-learning framework is proposed which is able to provide a personalized experience to the learner by recommending him study material according to his requirements, goals and calibre. A fuzzy logic based recommender agent framework is used to give further suggestions to learner to increase his/her satisfaction and provide enhanced and personalized learning experience. We also used the Matlab to simulate our recommender agent.
\end{abstract}

\section{Keywords}

Multi-agent, e-learning, fuzzy logic, personalization, recommender system.

\section{INTRODUCTION}

E-learning has been a revolution in recent years in the training field. It has been based onthe use of Information and Communication Technologies, and its most importantcharacteristic is offering distance learning and training to users based on their capability and requirements. The most effective way to enhance user's experience will be providing him a personalized learning environment based on his profile, future goals and learner feedbacks or reviews.

Web site personalization can be defined as the process of tailoring the content and structure of Web to the specific and individual needs of each user after accessing his personal requirements. Personalization of learning is one of the most important ways of improving the effectiveness of education. At the same time, it isalso a complex process that requires consideration of numerous factors such as learners' profiles, learning materials and learning strategies and goals.

Adaptive and collaborative e-learning systems can potentially deliver personalisedelectronic course material and services and are therefore able to accommodate a largevariety of learners. Developing such systems, however, are typically complex becausethey involve many dynamically interacting components, each with its own need forknowledge and information resources and the complexity of coordinating thecomponents.

The best way to provide learner with a customized learning experience with a proper recommendation system to further enhance his capabilities and attainment of his goals we propose a fuzzy based multi-agent recommender system for personalized e-learning.

Now what is an agent? An agent is a computer system that is situated in some environment, and that is capable of autonomous action in this environment in order to meet its design objectives. They are computational mechanism that exhibit a high degree of autonomy in performing actions in its environment based on the information (sensors, feedback)received from the environment.The agents have various characteristics- reactivity, autonomy, learning, cooperation, reasoning, communication, and mobility. The agents are able to perceive their environment and react to the situation based on their design objectives. The agents perceive their environment and communicate with each other in to work towards the solution to a problem. The agents are also an intelligent computer system. Therefore, they learn from their past experiences which help them in decision making in future situations.

A multi-agent system is one in which there are more than one agent, who interact with one another in a particular environment. Designing of a multi-agent system can be done by using a popular Prometheus development toolkit (PDT). The MAS using DSS has been entirely modelled by means of Prometheus methodology through Prometheus Design Tool. The tool provides the possibility of checking the consistency of thecreated system and of generating a skeleton code for JACK Intelligent Agentsdevelopment tool, as well as design reports in HTML.The process of creating a multi-system architecture, in accordance with thePrometheus methodology, consists of three phases, which are:

1. System specification, aimed to the identification of multiagent system entities, such as actors, system goals, scenarios, actions, percepts and roles.

2. High-level (architectural) design, which is centered in the description ofagent-role coupling, general system structure and interaction protocols.

3. Detailed design, in which each agent is described in detail in terms of capabilitiesevents, plans and data.

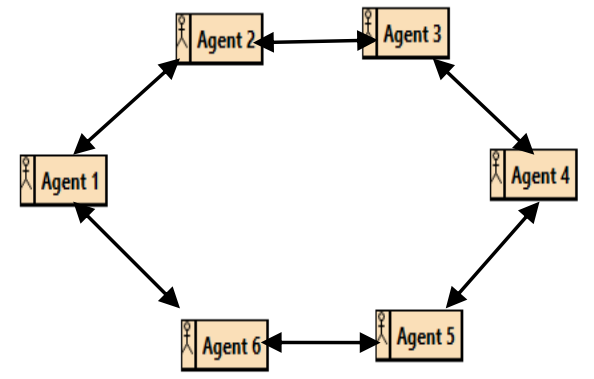

Fig 1: An example of multi agent system design using PDT. 


\section{LITERATURE SURVEY}

We studied various researches some of the major contributions for our proposed framework of a fuzzy based multi-agent recommender system for personalized e-learning are discussed in this section. A paper by Fuhua Lin, Peter Holt, Steve Leung andQin $\mathrm{Li}$ [1] presents architecture for developing adaptive-learning systems using intelligent agent technology and Web Services (WS)technology. They designedintelligent software agents for supporting users toaccomplish knowledge-intensive tasks. WS are designed for the integration ofdistributed knowledge and information resources and exposed as standard services using widely accepted protocols. Paper "A proposal of a multi-agent system for adapting learning content, user competence, context and mobile device" [2] proposed a new multi-agent system for adapting the learning contents according to the learner'scompetences, to the learner's context and to his/her mobile device. The paper also describesin detail the prototype developed for testing the proposed design.

E. Herrera-Viedma, A.G. L'opez-Herrera in their paper "A Review on Information Accessing Systems Based on Fuzzy Linguistic Modelling" [3] presents a survey of some fuzzy linguistic information access systems showinginformation retrieval systems, filtering systems, recommender systems, and web quality evaluation tools, which are based on tools of fuzzy linguistic modelling.

We analysed the concept and importance of personalization in the paper "Building a fuzzy knowledge management system for personalized e-learning" [4]. It presented The Fuzzy Knowledge Management System (FKMS) for personalized learning. The main objectiveof the system is to offer the most appropriate learning materials to the learners by considering their knowledge level and otherlearning characteristics. The knowledge base of the system contains course and concepts ontologies, learners' profiles andlearning objects knowledge.

LivuiPanait [5] provided a broad survey of the cooperative multi-agent learning literature. He attempted to find a broad view that leads to a division of the work into two categories, each with its own specialissues: applying a single learner to discover joint solutions to multi-agent problems (team learning), orusing multiple simultaneous learners (concurrent learning).

There is an another interesting survey [6] that surveys expert systems (ES) development using a literature review and classification of articles from 1995 to 2004 exploring ES methodologies and applications that have been developed during this period. This paper surveys and classifiesES methodologies using the following eleven categories: rulebased systems, knowledge-based systems, neural networks, fuzzy ESs, object orientedmethodology, case-based reasoning, system architecture, intelligent agent systems, database methodology, modelling, and ontologytogether with their applications for different researches and problem domains.

Félix Castro, Alfredo Vellido, ÀngelaNebot, and Francisco Mugica [7] described taxonomy of e-learning problems to which Data Mining techniques have been applied. It also discussed Students' classification based on their learning performance; detection of irregular learning behaviours; elearning system navigation and their interaction optimization; clustering according to similar e-learning system usage; and systems' adaptability to students' needs and capacities.

"Incorporating Filtering techniques in a fuzzy linguistic multiagent model for information gathering on the web" [8] provides a new fuzzy linguistic multi-agent model that composes of two information filtering techniques in its structure: a content-based filtering agent and a collaborative filtering agent. These elements are introduced to increase the information filtering possibilities of multi-agent system on the Web toimprove its retrieval issues. We also studied a domain ontologythat is suitable for adaptive e-learning environments [10].This ontology describes thelearning objects that compose to form a course, the learners and their learningstyles. Itsa multi-agent e-learning system that supports pre-defining andre-examining students' learning styles during the course for better personalization such that the learning style of each learner can be identified automatically anddynamically.

Elpiniki I. Papageorgiouand Jose L. Salmeron [11] creates an understandingof the on fuzzy cognitive maps by providing a review of the most recent applicationsand trends on fuzzy cognitive maps (FCMs) over the pastdecade.There's another survey "Review study on Fuzzy Cognitive Maps and their applications during the last decade" [12] that also reviewed the most recentapplications and trends on fuzzy cognitive maps (FCMs) at thelast ten years. FCMs have emergedwith a considerable degree of applicability due to theirdynamic characteristics and learning methodologies which make themessential for modelling, analysis, prediction and decision makingtasks as they improve the performance of the systems.

\section{SYSTEM FRAMEWORK}

We propose a multi agent based e-learning framework which is able to provide a personalized experience to the learner by recommending him study material according to his requirements, goals and calibre. This recommendation module is based on the fuzzy logic. Fuzzy logic is a way to make machines more intelligent enabling them to reason in a fuzzy manner like humans [13].

Designing of the system should be done on a PDT tool to insure consistency of the proposed framework. We have divided our e-learning system in various agents that collaborate to provide personalization to learner:

3.1 User: User is the learner who has signed up for any course. Users enter their details, learning goals, if it is short term course or detailed long term course. If already registered user logs in then he already has his account and details in our knowledge database.

3.2 Knowledge Database: This consists of all the account details of various learners along with their reports that depicts their performance. It also consists of various user feedbacks regarding any course that they have taken up. Knowledge base also consists of various course structures and study material for learning.

3.3 Interface agent:interface agent communicates the user query tothe task agent, and filters the retrieved documents from task agent in order to give the user thoseones that better satisfy his/her needs.

3.4 Task agent: (generally one for the interface agent), communicates the terms of learner's query to the information agents, and get those documents from every information agent that better fulfils the query, analysing them and resolving the possible conflicts among the informationagents. 


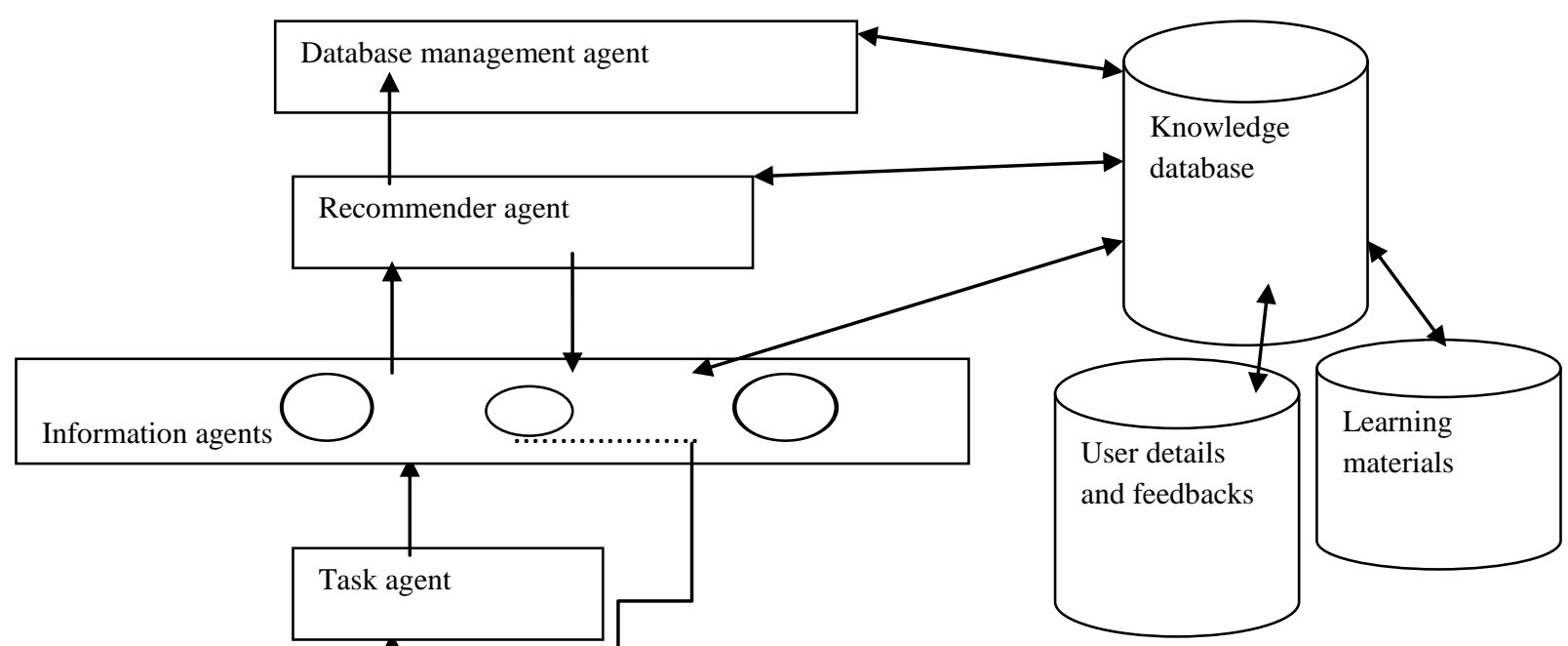

Fig 2: framework for e-learning system with multi-agents.

3.5 Information agents: which receive user query from the task agent and look for the documents in the information base.There can be multiple information agents that are specialized for various domains of courses. These agents collaborate to provide learning material to task agent.

3.6 Recommender agent: this agent is primarily responsible for recommending new learning material to the user depending upon two inputs: user feedbacks about various learning materials and learner report that denotes his performance and his goals attained so far.

3.7 Database management agent: this agent is responsible for managing knowledge database which can be broadly divided to two sections: first section consists of all the course structures and learning material, the other section consists of account details of all the users with their reports and various feedbacks.

Our proposed recommender agent uses fuzzy logic in his framework that enables him to recommend user new courses by taking two types of inputs:

(i) Learner performance: it can be graded as satisfactory or unsatisfactory.

(ii) User feedbacks: this consists of various user feedbacks about the various courses taken up by them. These inputs can be either poor, average, above average, good, very good and excellent.

Then user can select any particular course based on his interest and needs.

\section{ANALYSIS}

For recommender agent structure we need to use fuzzy logic. First step would be fuzzification, it is the process of decomposing a system input and/or output into one or more fuzzy sets. Here user feedback(X) fuzzy set will consist of six items and user performance will consist of two items(Y).

A fuzzy set is represented by a membership function defined on the universe of discourse. The universe of discourse is the space where the fuzzy variables are defined. The membership function gives the grade, or degree, of membership within the set, of any element of the universe of discourse. The membership function maps the elements of the universe onto numerical values in the interval $[0,1]$. A membership function value of zero implies that the corresponding element is definitely not an element of the fuzzy set, while a value of unity means that the element fully belongs to the set [13].

Now after obtaining fuzzy sets and defining the membership function we can apply fuzzy logic that interprets the final outcome. Fuzzy logic for our system can be stated like example:

if $\mathrm{Y}$ IS UNSATISFACTORY AND $\mathrm{X}$ IS GOOD THEN TRUE , if Y IS SATISFACTORY AND X IS EXCELLENT THEN FALSE.

Although there various other interpretations for fuzzy logicoperations, the following definitions are very convenient in embedded control applications:

$\operatorname{truth}(\mathrm{X}$ and $\mathrm{Y})=\operatorname{Min}(\operatorname{truth}(\mathrm{X}), \operatorname{truth}(\mathrm{Y}))$

$\operatorname{truth}(\mathrm{X}$ or $\mathrm{Y})=\operatorname{Max}(\operatorname{truth}(\mathrm{X}), \operatorname{truth}(\mathrm{Y}))$

$\operatorname{truth}(\operatorname{not} X)=1.0-\operatorname{truth}(X)$.

Now last part consists of defuzzification process, Defuzzification is such inverse transformation which maps the output from the fuzzy domain back into the crisp domain. Some of the defuzzification methods are:

Center-of-Area (C-o-A) - The C-o-A method refers to the Center-of-Gravity method because it computes the centroid of the composite area representing the output fuzzy term.

Center-of-Maximum (C-o-M) - In the C-o-M method only the peaks of the membership functions are used. The defuzzified crisp compromise value is determined by finding the place where the weights are balanced. The crisp output is computed as a weighted mean of the term membership maxima, weighted by the inference results. 
Mean-of-Maximum (M-o-M) -This method is used whenever the maxima of the membership functions are not unique.

We have Matlab simulator to map the two fuzzy sets, snapshots of the system are as followed:

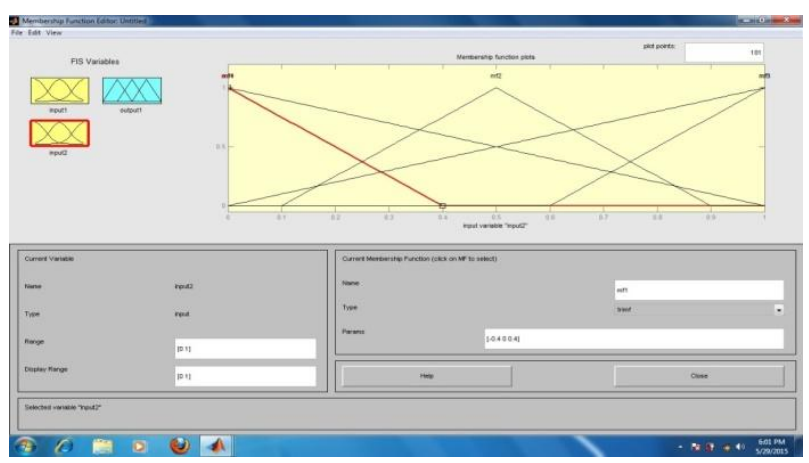

Fig 3: snapshot of the fuzzy set input : user performance

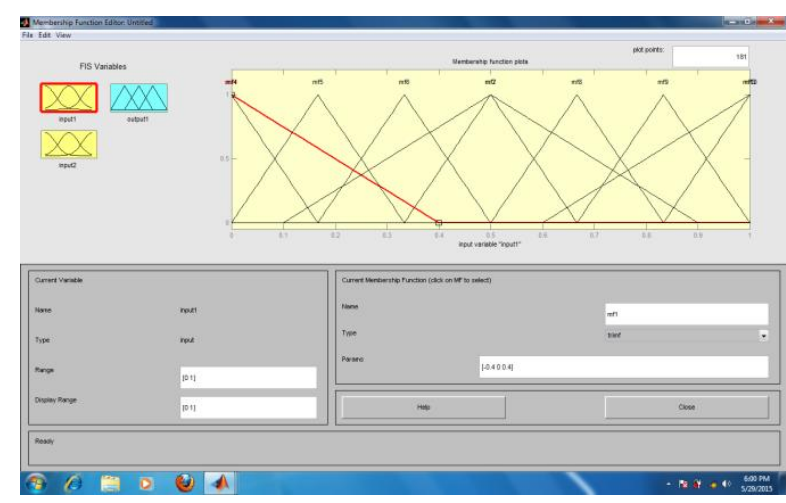

Fig 4: snapshot of the fuzzy set input : user feedback

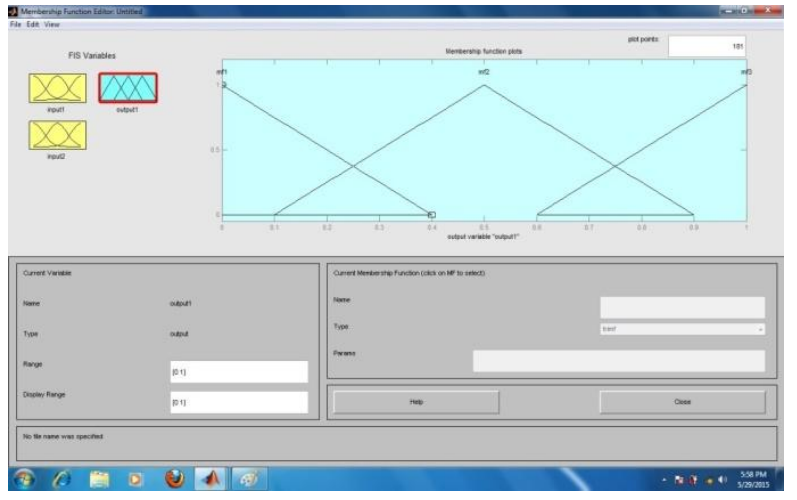

Fig 5: snapshot of the membership function: centre of maximum.

\section{CONCLUSION AND FUTURE SCOPE}

This paper aimed to provide a personalized learning experience in e-learning system by using multi-agent framework to deliver personalised electronic course material and services and are therefore able to accommodate a large variety of learners. To make our recommender system more efficient we proposed the use of fuzzy logic mapping to ensure learners attain their goals and get satisfactory results in desired time.

We can further make use of fuzzy cognitive maps (FCMs) to provide supervised in learning fuzzy-neural systems, when increasingly, data are available to model the problem, and the system becomes better at adapting itself and reaching a solution.

\section{REFERENCES}

[1] Fuhua Lin, Peter Holt, Steve Leung and Qin Li , 2006. A multiagent and service-oriented architecture for developing adaptive e-learning systems, Systems Int. J. Cont. Engineering Education and Lifelong Learning, Vol. 16, Nos. 1/2, 2006.

[2] Antonio GARCIA-CABOT, 2013. A proposal of a multiagent system for adapting learning contents to user competences, context and mobile device, www.mtf.stuba.sk/docs/doc/casopis_Vedecke_prace/32S N/004_Garcia-Cabot.2013

[3] E. Herrera-Viedma, A.G. L'opez-Herrera, 2010.A Review on Information Accessing Systems Based on Fuzzy Linguistic Modelling, International Journal of Computational Intelligence Systems, Spain. Vol.3, No. 4 (October, 2010), 420-437.

[4] Mehmet Ali Salahlia ,MuzafferOzdemir a, CumaliYasar, 1982. Building a fuzzy knowledge management system for personalized elearning,Procedia - Social and Behavioral Sciences 46 ( 2012 ) 1978 - 1982.

[5] LivuiPanait, Sean Luke, 2005. Cooperative Multi-Agent Learning: The State of the Art, Springer: Autonomous Agents and Multi-Agent Systems, 11, 387-434, 2005.

[6] Shu-Hsien Liao, 2004. Expert system methodologies and applications - a decade review from 1995 to 2004, .Science direct: Expert Systems with Applications, (2004) 1-11.

[7] Félix Castro, Alfredo Vellido, ÀngelaNebot, and Francisco Mugica,, 2007. Applying Data Mining Techniques to eLearning Problems, Springer: Applying Data Mining Techniques to e-Learning Problems, Studies in Computational Intelligence (SCI) 62, 183-221 (2007).

[8] E. Herrera-Viedmaa, F. Herreraa, L. Mart Onezb, J.C. Herreraa, A.G. Lopez, 2004.Incorporating Filltering techniques in a fuzzy linguistic multi-agent model for information gathering on the web, Science direct: Fuzzy Sets and Systems 148 (2004) 61-83.

[9] International journal of artificial intelligence and interactive multimedia. ISSN: 1989-1660 - VOL. II, NUMBER 1.

[10] Pham Quang Dung and Adina Magda Florea, 2013. Adaptation To Learning Styles In A Multi-Agent ELearning System, Internet Learning, Volume 2, Number 1, Spring 2013

[11] Elpiniki I. Papageorgiou and Jose L. Salmeron, 2013. A Review of Fuzzy Cognitive Maps ResearchDuring the Last Decade,IEEE Transactions on fuzzy systems, Vol. 21, No. 1, Februaury 2013.

[12] Elpiniki I. Papageorgiou, 2011.Review study on Fuzzy Cognitive Maps and theirapplications during the last decade, IEEE International Conference on Fuzzy Systems, June 27-30, 2011, Taipei, Taiwan.

[13] Marcelo Godoy Simoes, Introduction to Fuzzy Control.http://inside.mines.edu/ msimoes/documents/Int ro_Fuzzy_Logic. 